\title{
COMMUTATIVE CONSERVATION LAWS FOR GEODESIC FLOWS OF METRICS ADMITTING PROJECTIVE SYMMETRY
}

\author{
PETER TOPALOV
}

\begin{abstract}
We prove that the geodesic flow of a pseudo-Riemannian metric $g$ that admits a "nontrivial" projective symmetry $X$ is completely integrable. Nontriviality condition of the projective symmetry is expressed in the terms of the invariants of the pair forms $g$ and $L_{X} g$, where $L_{X}$ denotes the Lie derivative with respect to the vector field $X$. The theorem we propose can be considered as a "commutative" analog of the Noether theorem.
\end{abstract}

\section{Introduction}

Let $g$ be a pseudo-Riemannian metric given on the smooth manifold $M^{n}$. In what follows we call pseudo-Riemannian metrics simply metrics.

By definition, the projective symmetries of the pseudo-Riemannian manifold $\left(M^{n}, g\right)$ are vector fields that preserve the (unparameterized) geodesics of the metric $g$ (see Definition 1). The theory of the projective symmetries goes back to the classical works of Dini, Lie, Fubini, Weyl, Eisenhart, Cartan, Petrov and many others. The problem of the existence of conservation laws (integrals) of the geodesic flows of the metrics admitting projective symmetry and their relations with the general theory of relativity was considered by Davis and Moss in [3]. There was found one canonically given integral (see Remark 1). In the present paper we give a whole family of pairwise commuting integrals of the geodesic flows of the metrics admitting projective symmetry (Theorem 1). If some nondegeneracy condition is satisfied we prove the complete integrability of these flows. Our theorem lies in the same direction as the Noether theorem, i.e., the symmetries give conservation laws. Moreover, it appears that the projective symmetries give rise to commutative conservation laws.

The simplest variant of the Noether theorem asserts that provided the metric $g$ admits a Killing vector field, i.e., a smooth vector field $X$ whose one-parameter group of local diffeomorphisms $\left\{G_{X}^{t}\right\}_{t}$ preserves the metric $g$, then the linear in velocities function $I_{X}(\xi) \stackrel{\text { def }}{=} g(X, \xi)\left(\xi \in T M^{n}\right)$ is a first integral of the geodesic flow of the metric $g$. Moreover, it can be easily seen that if the manifold $M^{n}$ is connected and the vector field $X$ is not identically equal to zero on $M^{n}$, then

Received August 13, 2001.

Partially supported by MESC Grants no. MM-810/98 and 1003. 
the functions $E_{g}(\xi) \stackrel{\text { def }}{=} \frac{1}{2} g(\xi, \xi)$ and $I_{X}(\xi)$ are functionally independent on the whole tangent bundle $T M^{n}$.

Any Killing vector field $X$ of the metric $g$ preserves the geodesics, i.e., if $s \rightarrow \gamma(s) \in M^{n}$ is a geodesic of the metric $g$ then the curve $s \rightarrow G_{X}^{t}(\gamma(s))$ is also a geodesic of $g$. Of course, in the case of Killing symmetry, the parameter $s$ is natural. Recall that by definition a smooth curve $s \rightarrow \gamma(s)$ is written in natural parameter iff the length of its tangent field $\dot{\gamma}(s)$ is equal to some constant, $g(\dot{\gamma}, \dot{\gamma})=$ const.

Let us consider a "symmetry" $X$ that preserves the geodesics but only as sets of points on $M^{n}$, i.e., if $s \rightarrow \gamma(s)$ is a geodesic then $s \rightarrow G_{X}^{t}(\gamma(s))$ is also a geodesic but possibly not written in natural parameter.

Definition 1. A vector field $X$ is called projective symmetry of the metric $g$ iff its one-parameter group of local diffeomorphisms $\left\{G_{X}^{t}\right\}_{t}$ takes geodesic lines to geodesic lines (up to a change of the parameterization of the geodesic lines).

Let $X$ be a projective symmetry of $g$. Define the tensor field $b=b(g, X) \stackrel{\text { def }}{=}$ $L_{X} g$, where $L_{X}$ denotes the Lie derivative with respect to the vector field $X$, and consider the endomorphism of the tangent bundle $B=B(g, X) \stackrel{\text { def }}{=}\left(g^{i k} b_{k j}\right)$. Provided that $C$ is an endomorphism of the tangent bundle $T M^{n}$ and $x$ is a point of $M^{n}$, denote by $r_{C}(x)$ the degree of the minimal polynomial of the operator $\left.C\right|_{x}$.

Definition 2. The number $r \stackrel{\text { def }}{=} \max _{x \in M^{n}} r_{B}(x)$ is called first approximation rank of the projective symmetry $X$.

Consider the one-parameter family of quadratic forms

$$
K_{c}(g, X)(\xi) \stackrel{\text { def }}{=} \operatorname{det}(L+c \mathbf{1}) g\left((L+c \mathbf{1})^{-1} \xi, \xi\right), \quad \xi \in T M^{n},
$$

where $L=L(g, X) \stackrel{\text { def }}{=} B-\frac{\operatorname{tr} B}{n+1} \mathbf{1}$. It is clear that

$$
K_{c}(g, X)(\xi)=I_{n-1}(g, X)(\xi) c^{n-1}+\ldots+I_{0}(g, X)(\xi),
$$

where the quadratic forms $I_{k}(g, X)$ are globally defined on $M^{n}$ and $I_{n-1}(\xi)=$ $g(\xi, \xi)$.

The aim of the present note is to prove the next theorem.

Theorem 1. Suppose that the manifold $M^{n}$ is connected and let the metric $g$ admits a projective symmetry $X$ of first approximation rank $r$. Then the geodesic flow of the metric $g$ admits $r$ functionally independent integrals in involution $P_{1} \stackrel{\text { def }}{=} I_{n-1}(g, X), \ldots, P_{r} \stackrel{\text { def }}{=} I_{n-r}(g, X)$. For every fixed real constant $c$ the quadratic form $K_{c}(g, X)$ is a linear combination of the forms $P_{1}, \ldots, P_{r}$, i.e., there exist constants $\alpha_{1}, \ldots, \alpha_{r}$ such that $K_{c}=\sum_{k=1}^{r} \alpha_{k} P_{k}$.

We use the Legendre transformation $\mathrm{FL}_{g}: T M^{n} \rightarrow T^{*} M^{n}$ corresponding

to the metric $g$ and consider the symplectic form $\omega_{g} \stackrel{\text { def }}{=} \mathrm{FL}_{g}^{*} \omega$ on $T M^{n}$ where 
$\omega$ denotes the canonical symplectic structure on $T^{*} M^{n}$. The involutivity of the forms $P_{1}, \ldots, P_{r}$ in Theorem 1 is presumed with respect to the symplectic structure $\omega_{g}$.

Remark 1. It can be easily checked that $I_{n-2}(g, X)=2 \frac{\operatorname{tr} B}{n+1} g-b$. This integral is known. It was found by Davis and Moss in [3] ([4], Part II, Chapter II).

Using Theorem 1 we obtain the next corollaries.

Corollary 1. If the metric $g$ admits a projective symmetry of first approximation rank $n$ then its geodesic flow is completely integrable.

Corollary 2. The set $\Sigma$, of the points $x \in M^{n}$ such that $r(x)=r$, is open and dense in $M^{n}$.

A vector field $X$ is called homothetic symmetry of $g$ iff $L_{X} g=$ const $g$. It is clear that the homothetic symmetries are projective symmetries.

Remark 2. The first approximation rank of every homothetic symmetry is equal to one. The integrals given by Theorem 1 coincide with the "energy" integral.

Corollary 3. Any projective symmetry of first approximation rank 1 corresponds to a homothetic vector field. Provided the manifold $M^{n}$ is compact, any projective symmetry of first approximation rank 1 corresponds to a Killing vector field.

Now, assume that the vector field $X$ is complete. Then the local diffeomorphisms $\left\{G_{X}^{t}\right\}_{t}$ are globally defined on $M^{n}$ for all real values of the parameter $t$. Applying the results of [6] we obtain the next theorem.

Theorem 2. Suppose that the manifold $M^{n}$ is connected and let the geodesic flow of the metric $g$ be complete. Consider the one-parameter family of endomorphisms of the tangent bundle $B_{t} \in \Gamma\left(\operatorname{End}\left(T M^{n}\right)\right),\left(B_{t}\right)_{j}^{i} \stackrel{\text { def }}{=} g^{i \alpha}\left(\bar{g}_{t}\right)_{\alpha j}$, where $g_{t} \stackrel{\text { def }}{=}\left(G_{X}^{t}\right)^{*} g$. Define the number $R \stackrel{\text { def }}{=} \max _{x \in M^{n}} \max _{t \in \mathbf{R}} r_{B_{t}}(x)$. Then the geodesic flow of the metric $g$ admits $R$ functionally independent integrals in involution.

Remark 3. Due to the theorem of Liouville-Arnold, the existence of commutative integrals of a Hamiltonian system is useful for the integration of the corresponding equations. In this sense, Theorem 2 is not helpful because it doesn't give an explicit form of the corresponding integrals. To write these integrals explicitly we need to know the solutions $\left\{G_{X}^{t}\right\}_{t}$. Alternatively, the commutative integrals given by Theorem 1 are written only in the terms of the metric $g$ and the Lie derivative $L_{X} g$.

We will investigate the relations between the integrals of theorems 1 and 2 in a next paper. 


\section{Proof of the statements}

Proof of Theorem 1. The next well-known lemma is needed for the sequel (see [1], $\S 68$, and [2]).

Lemma 1. The vector field $X$ is a projective symmetry of the metric $g$ iff there exists a globally defined on $M^{n} 1$-form $\lambda$ such that

$$
\nabla_{k} b_{i j}=2 \lambda_{k} g_{i j}+\lambda_{i} g_{j k}+\lambda_{j} g_{i k}
$$

where $b_{i j}$ are the components of the form $b(g, X), \lambda_{i}$ are the components of $\lambda$, and $\nabla$ denotes the Levi-Civita connection corresponding to the metric $g$.

It follows from equation (3) that the 1 -form $\lambda$ is exact, i.e., there exists a globally defined on $M^{n}$ smooth function $\Lambda$ such that $\lambda_{k}=\frac{\partial \Lambda}{\partial x^{k}}$. Indeed, equation (3) shows that

$$
B_{j, k}^{i}=2 \lambda_{k} \delta_{j}^{i}+\lambda^{i} g_{j k}+\lambda_{j} \delta_{k}^{i},
$$

where $B_{j}^{i}$ are the components of the operator $B=B(g, X)$ and $\lambda^{i} \stackrel{\text { def }}{=} g^{i p} \lambda_{p}$. Contracting the indices $i$ and $j$ in the last formula, we obtain that $\lambda_{k}=\frac{\partial \Lambda}{\partial x^{k}}$ where $\Lambda=\frac{\operatorname{tr} B}{2(n+1)}$. Consider the tensor field

$$
a_{i j} \stackrel{\text { def }}{=} b_{i j}-2 \Lambda g_{i j}
$$

A direct calculation shows that

$$
\nabla_{k} a_{i j}=\lambda_{i} g_{j k}+\lambda_{j} g_{i k}
$$

Recall that two metrics $g$ and $\bar{g}$ are called geodesically equivalent iff they have the same geodesics (considered as unparameterized curves in the configuration space). In coordinates, the condition that the metrics $g$ and $\bar{g}$ have the same geodesics is equivalent to the next equation on the "deformation" tensor corresponding to the Levi-Civita connections of the metrics $g$ and $\bar{g}$

$$
\bar{\Gamma}_{j k}^{i}-\Gamma_{j k}^{i}=\phi_{j} \delta_{k}^{i}+\phi_{k} \delta_{j}^{i}
$$

where $\bar{\Gamma}_{j k}^{i}$ and $\Gamma_{j k}^{i}$ are the Christoffel symbols of the metrics $g$ and $\bar{g}$ respectively, $\delta_{j}^{i}$ is the Kronecker delta, and $\phi_{i}$ are the components of a globally defined 1-form (see [1]).

The statement of the next lemma is proved (under the condition of "nontriviality" of the geodesic equivalence) in [7]. A geodesic equivalence is said to be "non-trivial" if the covector field $\phi_{k}$ is not identically equal to zero. The proof proposed in [7] passes also in our case.

\section{Lemma 2.}

1) Suppose that the pseudo-Riemannian metrics $g$ and $\bar{g}$ are geodesically equivalent; then the tensors $a_{i j}$ and $\lambda_{i}$

$$
a_{i j} \stackrel{\text { def }}{=} A_{i}^{\alpha} g_{\alpha j}
$$




$$
\lambda_{i} \stackrel{\text { def }}{=}-A_{i}^{\alpha} \phi_{\alpha}
$$

where $(n+1) \phi_{i}=\frac{1}{2} \partial_{i} \ln \left|\frac{\operatorname{det} \bar{g}}{\operatorname{det} g}\right|$ and

$$
A_{j}^{i}(g, \bar{g}) \stackrel{\text { def }}{=}\left|\frac{\operatorname{det} \bar{g}}{\operatorname{det} g}\right|^{\frac{1}{n+1}} \bar{g}^{i \alpha} g_{\alpha j},
$$

satisfy the equation

$$
\nabla_{k} a_{i j}=\lambda_{i} g_{j k}+\lambda_{j} g_{i k}
$$

where $\nabla$ is the Levi-Civita connection corresponding to the metric $g$.

2) Conversely, if a non-degenerate symmetric tensor field $a_{i j}$ and an 1-form $\lambda_{i}$ satisfy equation (7), then the metric

$$
\bar{g}_{i j} \stackrel{\operatorname{def}}{=}\left(\frac{\operatorname{det} \hat{g}}{\operatorname{det} g}\right) \hat{g}_{i j},
$$

where $\hat{g}_{i j} \stackrel{\text { def }}{=} g_{i \alpha} a^{\alpha \beta} g_{\beta j}$, is geodesically equivalent to $g$.

Let us take an open set $U \subset M^{n}$ with compact closure in $M^{n}$. There exists a real constant $c_{0}$ such that the tensor field $a_{0} \stackrel{\text { def }}{=} a+c_{0} g$ is non-degenerate in $U$. It follows from (5) that $a_{0}$ satisfies the conditions of the second part of Lemma 2. Hence, the metrics $g$ and

$$
\begin{aligned}
\bar{g}_{0} & \stackrel{\text { def }}{=} \frac{\operatorname{det} \hat{g}_{0}}{\operatorname{det} g} \hat{g}_{0} \\
& =\frac{1}{\operatorname{det}\left(L+c_{0}\right)} g\left(L+c_{0} \mathbf{1}\right)^{-1},
\end{aligned}
$$

where $L_{j}^{i} \stackrel{\text { def }}{=} g^{i \alpha} a_{\alpha j}$, are geodesically equivalent in $U$. It follows from Theorem 1 in [6] that the one-parameter family of quadratic forms

$$
K_{\alpha}\left(g, \bar{g}_{0}\right) \stackrel{\text { def }}{=} \operatorname{det}\left(A\left(g, \bar{g}_{0}\right)+\alpha \mathbf{1}\right) g\left(A\left(g, \bar{g}_{0}\right)+\alpha \mathbf{1}\right)^{-1},
$$

where $A \stackrel{\text { def }}{=} A\left(g, \bar{g}_{0}\right)$ is given by formula (6), are pairwise commuting integrals of the geodesic flow of the metric $g$. A direct calculation shows that $A\left(g, \bar{g}_{0}\right)=$ $L+c_{0} \mathbf{1}$. Therefore, the one-parameter family of quadratic forms

$$
K_{c}(g, X) \stackrel{\text { def }}{=} \operatorname{det}(L+c \mathbf{1}) g(L+c \mathbf{1})^{-1},
$$

where $L \stackrel{\text { def }}{=} g^{-1} a=g^{-1}(b-2 \Lambda g)=B-2 \Lambda \mathbf{1}$, are pairwise commuting integrals of the geodesic flow of the metric $g$ on $U$. The forms $K_{c}(g, X)$ are globally defined on $M^{n}$ and the open set $U$ is taken arbitrary. Therefore, the forms $K_{c}(g, X)$ are pairwise commuting integrals of the geodesic flow of the metric $g$. Finally, the statement of Theorem 1 follows from Theorem 2 in [6] (see also Remark 2 there) and the fact that the degrees of the minimal polynomials of the operators $\left.A\left(g, \bar{g}_{0}\right)\right|_{x}$ and $\left.B\right|_{x}\left(x \in M^{n}\right)$ coincide. Theorem 1 is proved.

Corollary 1 follows directly from Theorem 1. 
Proof of Corollary 2. It follows from Theorem 1 that the quadratic forms $P_{1}, \ldots, P_{r}$ are functionally independent on $T M^{n}$. By definition, $r_{B}(x) \leq r$. Suppose that there exists a connected open set $U \subset M^{n}$ such that for every $x \in U, r_{B}(x)<r$. Hence, $r_{0} \stackrel{\text { def }}{=} \max _{x \in U} r_{B}(x)<r$. Applying Theorem 1 to the manifold $\left(U,\left.g\right|_{U}\right)$, we obtain that there exist constants $\alpha_{1}, \ldots, \alpha_{r_{0}}$ such that $P_{r_{0}+1}=\sum_{k=1}^{r_{0}} \alpha_{k} P_{k}$ on $T U$. The last equality gives that the quadratic forms $P_{1}, \ldots, P_{r}$ are not functionally independent on $T M^{n}$. This contradiction shows that $\Sigma$ is dense in $M^{n}$.

Let us prove that $\Sigma$ is open in $M^{n}$. It follows from Lemma 2 and Proposition 3 both proved in [6] that $r_{B}(x)=\operatorname{rk}\left\{\left.I_{n-1}(g, X)\right|_{x}, \ldots,\left.I_{0}(g, X)\right|_{x}\right\}$, where $\left.I_{k}(g, X)\right|_{x}$ are considered as elements of $\operatorname{Symm}\left(T_{x}^{*} M^{n} \otimes T_{x}^{*} M^{n}\right)$. Therefore, there exists an open neighborhood $V(x)$ of the point $x$ such that for every $y \in V(x)$ we have $r_{B}(y) \geq r_{B}(x)$. Taking $x \in \Sigma$, we obtain that $r_{B}(y)=r$. This completes the proof of Corollary 2.

Proof of Corollary 3. It is clear that the projective symmetry $X$ is of first approximation rank 1 if and only if $L_{X} g=f g$ where $f$ is a smooth function on $M^{n}$. It follows from Theorem 1 and formula (1) that $K_{c}(g, X)=(f /(n+1)+c)^{n-1} g$ is an integral of the geodesic flow of the metric $g$. Therefore, $f=$ const. This proves the first statement of Corollary 3.

Suppose that the manifold $M^{n}$ is compact and let $X$ be a vector field such that $L_{X} g=c g$ where $c$ is some constant. Denoting by $\sigma_{g}$ the volume element corresponding to the metric $g$, we obtain that $L_{X} \sigma_{g}=\frac{c n}{2} \sigma_{g}$. Integrating these $n$-forms, we obtain $0=\int_{M^{n}} L_{X} \sigma_{g}=\frac{c n}{2} \operatorname{Vol}_{g}\left(M^{n}\right)$. Therefore, $c=0$. Corollary 3 is proved.

Proof of Theorem 2. It is clear that the metrics $g$ and $\bar{g}_{t}$ are geodesically equivalent. Finally, the statement of Theorem 2 follows from Theorem 2 in [6].

2.1. Examples. Here we consider some examples that illustrate our results.

Example 1. Denote by $\left\{\left(x_{1}, x_{2}, x_{3}\right)\right\}$ the coordinates in $\mathbf{R}^{3}$ and consider the Lorenz metric

$$
d g_{1}^{2} \stackrel{\text { def }}{=} 4 A d x_{1} d x_{3}+d x_{2}^{2}+2 x_{1} d x_{2} d x_{3}+x_{1}^{2} d x_{3}^{2},
$$

where $A \stackrel{\text { def }}{=} x_{2}+a x_{3} \neq 0, a=$ const. The vector field

$$
X_{1} \stackrel{\text { def }}{=} x_{3}\left(2 x_{2}+a x_{3}\right) \frac{\partial}{\partial x_{2}}+x_{3}^{2} \frac{\partial}{\partial x_{3}}
$$

is a projective symmetry of the metric $g$ (see [9]). A direct calculation shows that

$$
L\left(g_{1}, X_{1}\right)=\left[\begin{array}{ccc}
x_{3} & 1 & x_{1} \\
0 & x_{3} & 2 A \\
0 & 0 & x_{3}
\end{array}\right] .
$$

We have that $\left(L-x_{3} \mathbf{1}\right)^{2}=2 A \neq 0$ and $\left(L-x_{3} \mathbf{1}\right)^{3} \equiv 0$. Therefore the first approximation rank of this symmetry is $r=3$. The integrals in involution $P_{1}$, 
$P_{2}$ and $P_{3}$ given by Theorem 1 are functionally independent, and therefore the geodesic flow of the metric $g$ is completely integrable. Remark that till now only the integrals $P_{1}$ (the "energy" integral) and $P_{2}$ have been known. It is interesting that the quadratic forms $\left.P_{1}\right|_{x},\left.P_{2}\right|_{x}$ and $\left.P_{3}\right|_{x}$ are not simultaneously diagonalizable on the tangent plane $T_{x} \mathbf{R}^{3}$.

Example 2. Consider the Lorentz metric in $\mathbf{R}^{4}=\left\{\left(x_{1}, x_{2}, x_{3}, x_{4}\right)\right\}$

$$
d g_{2}^{2} \stackrel{\text { def }}{=} 2 d x_{1} d x_{2}+x_{1}^{2} \mathcal{P}\left(d x_{3}, d x_{4}\right), x_{1} \neq 0,
$$

where the coefficients $P_{\alpha \beta}\left(P_{\alpha \beta}=P_{\beta \alpha}\right)$ of the positive definite quadratic form $\mathcal{P}\left(d x_{3}, d x_{4}\right) \stackrel{\text { def }}{=} \sum_{\alpha, \beta=3}^{4} P_{\alpha \beta}\left(x_{3}, x_{4}\right) d x_{\alpha} d x_{\beta}$ depend only on the variables $x_{3}$ and $x_{4}$. The vector field

$$
X_{2} \stackrel{\text { def }}{=} x_{1} x_{2} \frac{\partial}{\partial x_{1}}+x_{1}^{2} \frac{\partial}{\partial x_{2}}
$$

is a projective symmetry of the metric $g$ (see [10]). It can be easily checked that the first approximation rank of the projective symmetry $X_{2}$ is $r=3$. Therefore, the geodesic flow of the metric $g$ admits 3 functionally independent integrals in involution.

The metrics given in Examples 1 and 2 are not flat. An infinitesimal variant of the construction proposed in [6], Sect. IV, allows constructing projective symmetries of first approximation rank $n$ of the standard flat metrics in $\mathbf{R}^{n}$. Applying Theorem 1 we obtain complete families of pairwise commuting integrals of these metrics.

\section{References}

[1] L. Eisenhart, Riemannian Geometry, Princeton University Press, 1960.

[2] A. Solodovnikov, Projective transformations of Riemannian spaces, Uspehi Mat. Nauk (N.S.) 11 (1956), no. 4 (70), 45-116.

[3] W. Davis, M. Moss, Conservation laws of the general theory of relativity. II: Space-times admitting certain symmetry properties more general than motions, Nuovo Cimento (10) 381965 1558-1569.

[4] W. Davis, Classical fields, particles, and the theory of relativity, Gordon and Breach, 1970.

[5] N. Sinyukov, Geodesic mappings of Riemannian spaces, Nauka, Moscow, 1979.

[6] P. Topalov, Geodesic hierarchies and involutivity, J. Math. Phys. 42 (2001) no. 8, 38983914.

[7] N. Sinyukov, To the theory of geodesical mappings, Soviet Math. Docl. 169 (4) (1966), $770-772$.

[8] N. Sinyukov, An invariant transformation of Riemannian manifolds with common geodesics, Soviet Math. Docl. 137 (6) (1961), 1312-1314.

[9] A. Aminova, Lie algebras of infinitesimal projective transformations of Lorentz manifolds, Russian Math. Surveys 50 (1995), no. 1, 69-143.

[10] G. Hall, D. Lonie, Projective collineations in spacetimes, Classical Quantum Gravity 12 (1995), no. 4, 1007-1020. 
Institut Für Mathematik, Universität Zürich, CH-8057 Zürich, Switzerland.

Department of Differential Equations, Institute of Mathematics, BAS, Sofia, Bulgaria.

E-mail address: topalov@banmatpc.math.bas.bg 